\title{
AVALIAÇÕES HISTOLÓGICA E HISTOMORFOMÉTRICA DE TESTÍCULOS DE BOVINOS COM DERMATITE DIGITAL
}

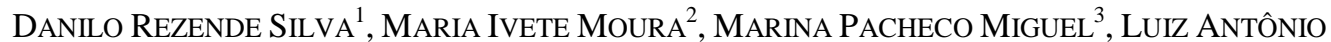 \\ Franco DA SILVA ${ }^{4}$, MOEMA PACHECO CHEDIAK MATOS ${ }^{4}$, VERIDIANA MARIA BRIANEZI DigNANI \\ MOURA $^{4}$ \\ ${ }^{1}$ Pós-graduando da Escola de Veterinária e Zootecnia da Universidade Federal de Goiás, Goiânia, GO, Brasil. \\ drs-patovet@hotmail.com \\ ${ }^{2}$ Pós-Doutoranda da Escola de Veterinária e Zootecnia da Universidade Federal de Goiás, Goiânia, GO, Brasil. \\ ${ }^{3}$ Professora Doutora da Universidade Federal de Goiás, Campus de Jataí, Jataí, GO, Brasil. \\ ${ }^{4}$ Professores Doutores da Escola de Veterinária e Zootecnia da Universidade Federal de Goiás, Goiânia, GO, \\ Brasil.
}

\begin{abstract}
A infecção da região digital é a causa mais comum de claudicação em bovinos, podendo levar à inutilização de animais de alta produção e destinados à reprodução. Este estudo teve como objetivo avaliar aspectos histológicos e histomorfométricos dos testículos de bovinos com dermatite digital. Foram analisadas diferentes porções dos testículos de 20 bovinos da raça Nelore, entre 25 e 30 meses, sendo 10 com de dermatite digital e 10 saudáveis. Todas as amostras testiculares avaliadas apresentaram algum grau de degeneração do epitélio tubular seminífero. Infiltrado inflamatório intersticial mononuclear foi
\end{abstract}

\section{RESUMO}

PALAVRAS-CHAVE: alterações testiculares; enfermidade podal; morfometria.

\section{TESTIS HISTOLOGIC AND HISTOMORPHOMETRIC EVALUATIONS OF CATTLE WITH DIGITAL DERMATITIS}

\section{ABSTRACT}

The infection of the digital area is the most common cause of lameness in cattle, leading to the rejection of highproduction animals destined to reproduction. This study aimed at histological and histomorphometric evaluations of the testis of bovines with digital dermatitis. Different portions of testis from 20 Nellore animals, ten healthy and ten with digital dermatitis, between 25 and 30 months of age, were analyzed. All analyzed testis showed some degree of degeneration of the seminiferous epithelium. Mononuclear interstitial infiltrate was observed in testis of observado em testículos de bovinos saudáveis e com dermatite digital. A altura do epitélio tubular seminífero de todas as regiões testiculares foi maior nos animais com dermatite digital, assim como foram observados maior área da luz tubular e maior diâmetro tubular nos testículos desses bovinos. Concluiu-se que bovinos jovens da raça Nelore apresentam diferentes graus de degeneração testicular, bem como orquite intersticial crônica inespecífica, sem relação com a dermatite digital. Ainda, as alterações histomorfométricas dos testículos desses bovinos não possuem relação com a enfermidade podal.

KEYWORDS: morphometry; podal disease; testicular changes.

both healthy bovines and the ones with digital dermatitis. Bovines with digital dermatitis presented higher height of seminiferous tubular epithelium in all regions of testis, larger area of the tubular lumen, and larger tubular diameter. We concluded that young Nellore bovines show different degrees of testicular degeneration, as well as chronic nonspecific interstitial orchitis, unrelated to digital dermatitis. The testis histomorphometric changes of these animals are not related with foot disease. 


\section{INTRODUÇÃO}

A dermatite digital é reconhecida como uma das principais enfermidades podais dos bovinos, sendo registrada em países como Austrália, Canadá, França, Alemanha, Israel e Japão. No Brasil foi descrita no estado de Goiás por LEÃO et al. (2009), que verificaram a frequência da dermatite digital nos municípios de Jataí e Orizona, e encontraram 256 animais enfermos. Trata-se uma epidermatite superficial difusa ou circunscrita do dígito, comprometendo a porção marginal da banda coronária e, comumente, a porção plantar, especificamente na parte mais elevada do espaço interdigital entre os bulbos do talão (DEMIRKAN et al., 2000).

BERGSTEN (1997) caracterizou a dermatite digital como uma inflamação superficial contagiosa da epiderme, próxima à margem coronária e ao espaço interdigital do estojo córneo bovino. Para CASTRO et al. (2008), a lesão instala-se mais comumente na região entre os talões e no espaço interdigital dorsal; no entanto, com menor frequência, as lesões podem ocorrer na região da comissura flexora do espaço interdigital.

Considerando as diferentes enfermidades podais, a infecção da região digital é a causa mais comum de claudicação em bovinos, podendo levar à inutilização de animais de alta produção e com finalidade reprodutiva (CHAPLIN et al., 2000). Nos bovinos destinados à reprodução, os efeitos decorrentes das afecções podais podem gerar dificuldades na monta. MOURA et al. (2010) estudaram a impotência coeundi em animais portadores de dermatite digital, cuja libido foi inferior em relação àqueles sem alterações podais. Além disso, afirmaram que bovinos tratados no início das lesões apresentam redução dos estímulos dolorosos, o que confere maior conforto, melhora do apetite e proporciona maior ganho de peso. Já bovinos com deficiência de energia, proteínas, minerais ou vitaminas podem ter todo o sistema reprodutivo comprometido (LEONEL et al., 2006).

A dificuldade de locomoção dos animais acometidos ocasiona baixa ingestão de alimento, chegando a comprometer $20 \%$ e $25 \%$ da produção de leite e de carne, respectivamente. Além disso, citam-se outros impactos econômicos negativos como infertilidade, queda na produtividade, alto custo com tratamentos e descarte precoce de animais de alto valor zootécnico (FARROW, 1999; FERREIRA et al., 2004; SILVA et al., 2004). Ainda, dores contínuas podem levar o animal ao estresse crônico, desencadeando uma resposta inibitória do organismo sobre o eixo hipotalâmico-hipofisário-gonadal (RAZDAN et al., 2001).

A biometria e a histometria do testículo são parâmetros que podem ser abordados na caracterização da puberdade e maturidade sexual, além de darem suporte aos estudos comparativos com animais de outras espécies (SILVA, 1995). O diâmetro do túbulo seminífero é um parâmetro que pode ser abordado como indicador da atividade espermatogênica em avaliações com objetivos experimentais e análises toxicológicas (ASSISNETO, 2003).

$\mathrm{O}$ volume do parênquima testicular de mamíferos é bastante variável, sendo um dos principais fatores responsáveis pela diferença observada na eficiência da produção espermática nas diversas espécies (FONTANA et al., 1990; HOCKING, 1997; FRANÇA \& GODINHO, 2003). A mensuração do peso testicular compreende método de avaliação indireta do desenvolvimento reprodutivo, enquanto a quantificação do epitélio seminífero representa um método direto de avaliar o estágio reprodutivo dos machos (VIU et al., 2006).

Diante das considerações acerca das enfermidades digitais e suas implicações sobre o sistema reprodutor dos bovinos, o presente estudo teve por objetivo realizar análises histológica e histomorfométrica de testículos bovinos da raça Nelore com dermatite digital.

\section{MATERIAL E MÉTODOS}

Foram utilizados 20 bovinos da raça Nelore, machos, não castrados, entre 25 e 30 meses, sendo 10 portadores de dermatite digital (G1) e 10 saudáveis (G2).

Para as avaliações histológica e histomorfométrica, fragmentos das porções proximal (CR), média (ME) e distal (CA) de cada testículo, obtidos a partir de orquiectomia, foram colhidos e fixados em formol tamponado a $10 \%$ durante 48 horas. A Figura 1 ilustra o casco de um bovino com dermatite digital e o padrão de colheita dos fragmentos testiculares. 


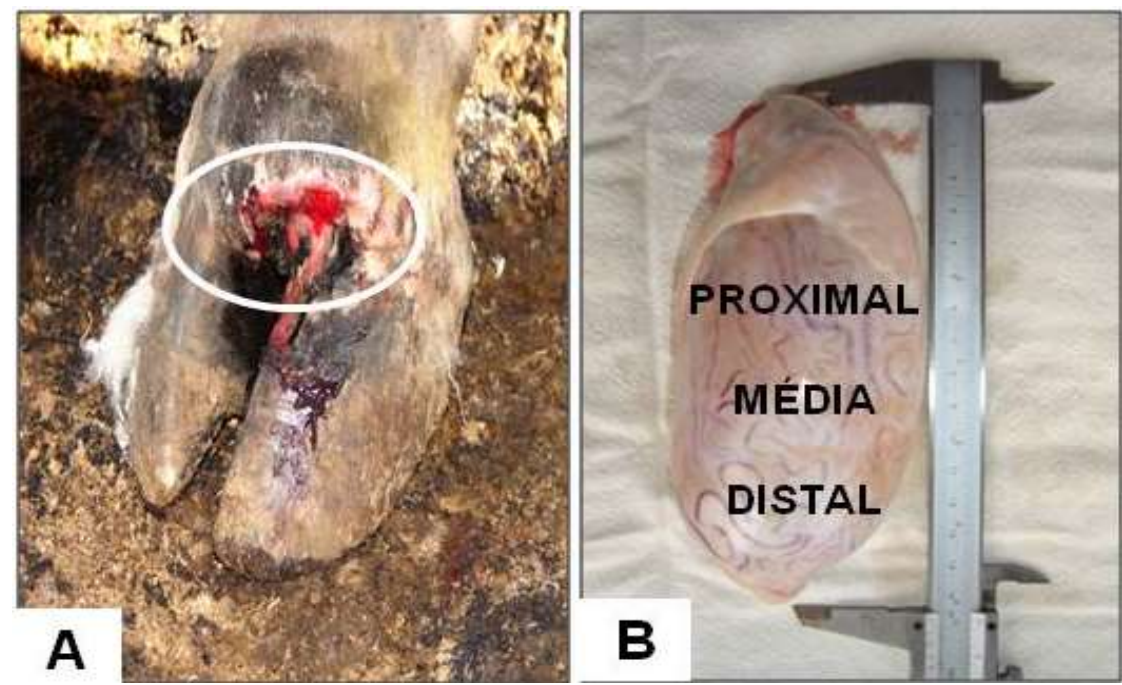

Figura 1. A) Casco bovino. Dígito com dermatite digital bovina (área circulada). B) Testículo bovino. Padrão de colheita das porções proximal, média e distal.

As amostras testiculares foram identificadas de acordo com o número do animal, a lateralidade do órgão (direito ou esquerdo) e a porção à qual pertencia, sendo então processadas, incluídas em parafina e cortadas em micrótomo rotativo, obtendose cortes de $3 \mu \mathrm{m}$, que foram distendidos sobre lâminas histológicas e corados pela técnica de hematoxilina e eosina (HE).

Para a análise histológica, os cortes foram avaliados em microscópico óptico nos aumentos de $5 \mathrm{x}, 10 \mathrm{x}$ e $20 \mathrm{x}$, considerando as seguintes alterações: degeneração vacuolar das células epiteliais dos túbulos seminíferos, infiltrado inflamatório intersticial e hipoplasia tubular. Para a degeneração tubular foram atribuídos escores de acordo com a intensidade da lesão, sendo (0) ausente, (1) discreta, (2) moderada e (3) acentuada. O infiltrado inflamatório intersticial foi classificado quanto ao tipo celular predominante (mononuclear ou polimorfonuclear) e quanto à intensidade (ausente, discreta, moderada e acentuada). A hipoplasia tubular foi avaliada considerando-se a ausência ou presença.

A análise histomorfométrica foi realizada com o auxílio de um sistema de análise digital de imagem, que consiste de uma videocâmara digital (Sony Cyber-Shot 3.2 MP, modelo DSC-P71), que transmite a imagem capturada do microscópio (Carl Zeiss@, modelo Jenaval, com objetivas planacromáticas) a um computador (Pentium®4, 3,20 GHz, 1GB de memória RAM, com placa digitalizadora e software Image $\mathrm{J}$ 1.3.1, NIH, Estados Unidos). Para as mensurações foram considerados o diâmetro tubular, a altura do epitélio seminífero e a área da luz tubular, medidas em micrômetros $(\mu \mathrm{m})$.
No cálculo da altura do epitélio foram consideradas as medidas das extremidades no mesmo túbulo em forma de cruz. Para a realização das medidas, foram escolhidos três túbulos seminíferos inteiros e que apresentassem morfologia em plano de corte transversal no campo de observação. O aumento 250x foi utilizado para as diferentes mensurações.

Para a análise estatística utilizou-se o programa GraphPad InStat (Version 3.05 for Windows). A partir do teste de normalidade de Kolmogorov-Smirnov, foram empregados os testes $t$ não-pareado (variáveis paramétricas) e Mann Whithney (variáveis não-paramétricas). Adotou-se o nível de significância de 5\% (p<0,05) (SAMPAIO, 1998).

O presente trabalho faz parte de um projeto maior, o qual foi submetido à Comissão de Ética em Pesquisa da Universidade Federal de Goiás, sendo protocolado sob o $\mathrm{n}^{\circ}$ 018, com parecer favorável.

\section{RESULTADOS}

A principal alteração nas células epiteliais dos túbulos seminíferos foi a degeneração vacuolar. Em muitas amostras, observou-se infiltrado inflamatório intersticial predominantemente mononuclear. Em nenhuma das amostras foi observada hipoplasia tubular.

No grupo de bovinos com dermatite (G1) foram analisadas 56 lâminas, já que um animal era monorquida e houve perda de uma amostra durante o processamento do material. Nesse grupo, 23 (41\%), $30(54 \%)$ e três (5\%) amostras apresentaram degeneração vacuolar do epitélio seminífero de intensidade discreta, moderada e acentuada, 
respectivamente.

No grupo de bovinos saudáveis (G2), do total de 60 amostras analisadas, 27 (45\%) apresentaram degeneração vacuolar discreta (Figura 2A), 27 (45\%) moderada (Figura 2B) e $6(10 \%)$ acentuada (Figura 2C).

No G1, infiltrado inflamatório mononuclear foi observado em $16(28,5 \%)$ amostras (Figura 2D) e polimorfonuclear em $2(3,6 \%)$. O restante das amostras não apresentou infiltrado inflamatório intersticial. No G2, dez (16,6\%) amostras apresentaram infiltrado inflamatório mononuclear, sendo que infiltrado inflamatório polimorfonuclear não foi observado em nenhuma das amostras.

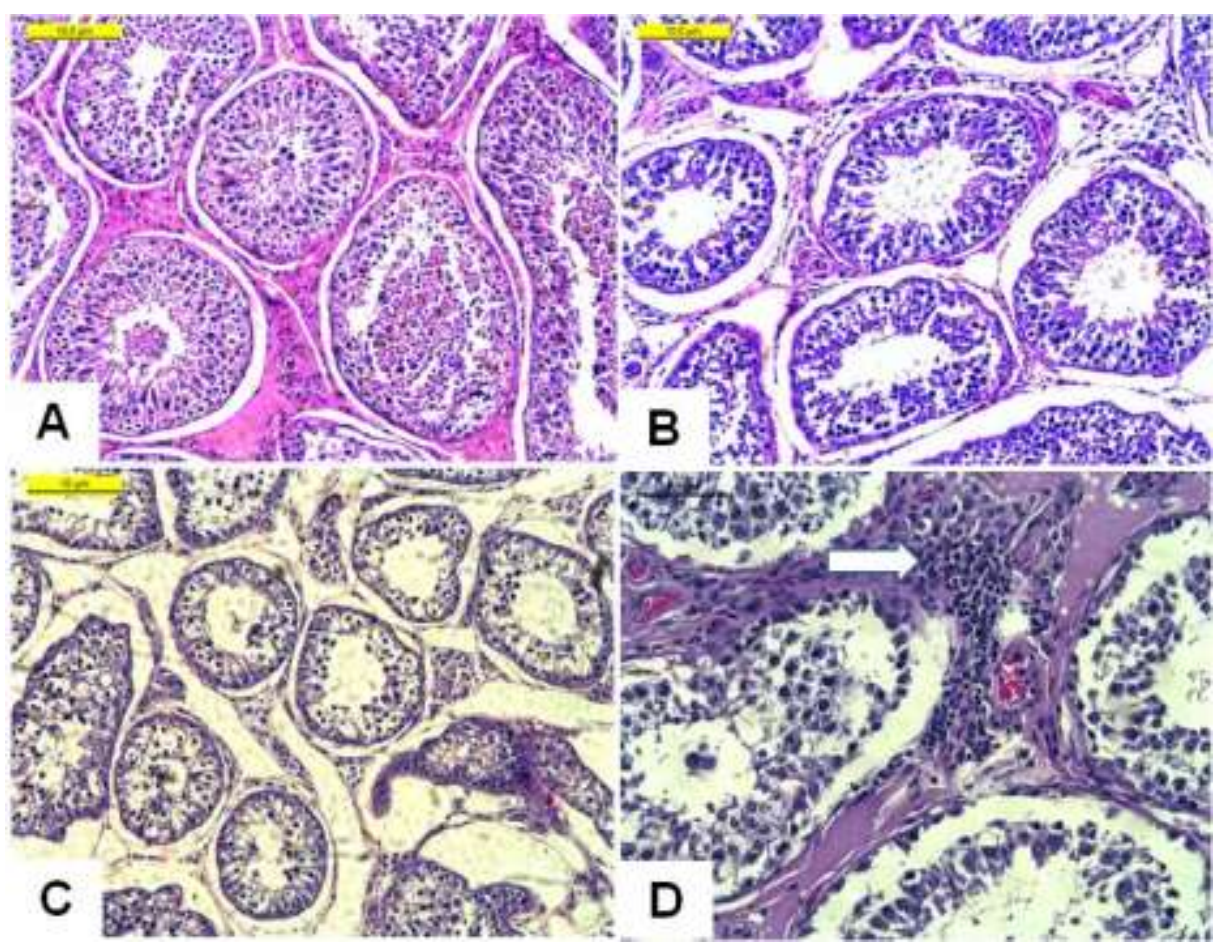

Figura 2. Fotomicrografia de tesículo de bovino. A, B e C) Túbulos seminíferos apresentando degeneração do epitélio em intensidades discreta, moderada e acentuada, respectivamente. HE, obj. 10x. D) Infiltrado inflamatório mononuclear intersticial (seta). HE, obj. 20x.

Não houve diferença entre os grupos estudados quanto à degeneração vacuolar. Contudo, ressalta-se que essa foi a alteração predominante nos testículos dos animais de ambos os grupos (Tabela $1)$.

A altura do epitélio tubular seminífero de todas as regiões foi diferente entre os grupos, sendo maior em G1. A área da luz tubular da porção distal direita dos testículos apresentou diferença significativa entre G1 e G2, sendo maior em G1. O diâmetro do túbulo seminífero distal direito foi estatisticamente diferente entre os dois grupos, sendo maior em G1.

Não foi observada diferença significativa entre as demais medidas, porém o diâmetro do túbulo apresentou valores maiores em G1 quando comparado a G2, e a área da luz tubular mostrou medidas menores em G2 (Tabela 2).

Tabela 1. Médias dos escores atribuídos à degeneração vacuolar (DV) do epitélio seminífero de acordo com as porções testiculares avaliadas nos grupos de bovinos com dermatite digital (G1) e saudáveis (G2)

\begin{tabular}{lcc}
\hline \multirow{2}{*}{ Variáveis } & \multicolumn{2}{c}{ Média } \\
\cline { 2 - 3 } & $\mathrm{G} 1$ & $\mathrm{G} 2$ \\
\hline DV - porção distal direita & 1.700 & 1.800 \\
DV - porção distal esquerda & 1.444 & 1.500 \\
DV - porção proximal direita & 1.300 & 1.400 \\
DV - porção proximal esquerda & 1.778 & 1.700 \\
DV - porção média direita & 1.889 & 1.600 \\
DV - porção média esquerda & 1.667 & 1.700 \\
\hline
\end{tabular}


Tabela 2. Médias das variáveis altura do epitélio seminífero, área da luz tubular e diâmetro tubular, para os grupos de bovinos com dermatite digital (G1) e saudáveis $(\mathrm{G} 2)$

\begin{tabular}{lcc}
\hline \multirow{2}{*}{ Variáveis } & \multicolumn{2}{c}{ Média } \\
\cline { 2 - 3 } & $\mathrm{G} 1$ & $\mathrm{G} 2$ \\
\hline Altura do epitélio da porção distal direita & $36.318^{\mathrm{a}}$ & $28.538^{\mathrm{b}}$ \\
Altura do epitélio da porção distal esquerda & $39.94^{\mathrm{a}}$ & $33.319^{\mathrm{b}}$ \\
Altura do epitélio da porção proximal direita & $39.626^{\mathrm{a}}$ & $32.478^{\mathrm{b}}$ \\
Altura do epitélio da porção proximal esquerda & $39.186^{\mathrm{a}}$ & $35.163^{\mathrm{b}}$ \\
Altura do epitélio da porção média direita & $39.883^{\mathrm{a}}$ & $31.971^{\mathrm{b}}$ \\
Altura do epitélio da porção média esquerda & $36.913^{\mathrm{a}}$ & $35.040^{\mathrm{b}}$ \\
Área luz da porção distal direita & $4831.0^{\mathrm{a}}$ & $3363.8^{\mathrm{b}}$ \\
Área luz da porção distal esquerda & 3794.6 & 3970.1 \\
Área luz da porção proximal esquerda & 3614.8 & 3990.0 \\
Área luz da porção proximal direita & 3482.5 & 3692.9 \\
Área luz da porção média direita & 3830.6 & 3747.5 \\
Área luz da porção média esquerda & 3837.5 & 3341.0 \\
Diâmetro da porção distal direita & $162.52^{\mathrm{a}}$ & $129.43^{\mathrm{b}}$ \\
Diâmetro da porção distal esquerda & 158.64 & 150.72 \\
Diâmetro da porção proximal direita & 157.45 & 137.69 \\
Diâmetro da porção proximal esquerda & 158.40 & 160.13 \\
Diâmetro da porção média direita & 161.90 & 150.58 \\
Diâmetro da porção média esquerda & 154.09 & 150.64 \\
\hline L & &
\end{tabular}

Letras diferentes na mesma linha diferem entre si $(\mathrm{p}<0,05)$.

\section{DISCUSSÃO}

$\mathrm{Na}$ avaliação histológica das amostras de testículo bovino, a presença de infiltrado inflamatório configura quadro de orquite intersticial, que pode ser resultado de diversas condições patológicas, como traumas, agentes infecciosos, granulomas espermáticos ou por extensão de cistites, uretrites, prostatites e epididimites (BOUCIF et al., 2011; BOUSMAHA \& KHOUDJA, 2012). De acordo com CHATE et al. (2009), a orquite primária normalmente é hematógena e o principal agente em bovinos reprodutores é a Brucella abortus. Porém, a brucelose causa orquite necrosante, o que não foi verificado nos animais do presente estudo.

Embora o infiltrado inflamatório mononuclear tenha sido observado em um número maior de amostras testiculares dos bovinos com dermatite, não é possível relacionar esse achado à enfermidade podal, já que, segundo LADDS \& FOSTER (2007), o acúmulo focal de leucócitos mononucleares é ocasionalmente encontrado nos testículos de várias espécies como achado acidental. Além disso, os mesmos autores afirmam que em testículos normais de touros são observados com frequência focos discretos de infiltrado de células mononucleares adjacentes a túbulos seminíferos. Ainda, COSTA et al. (2007), na avaliação de testículos de ovinos deslanados, perceberam que, no testículo direito, os túbulos seminíferos apresentavam atividade espermatogênica; contudo com variáveis estágios de degeneração. Nesse mesmo estudo, os autores encontraram células inflamatórias mononucleares entre os túbulos no espaço intersticial.

Com relação à degeneração vacuolar de células dos túbulos seminíferos observada em ambos os grupos, esta constitui uma alteração reversível, porém, dependendo da intensidade e do tempo de ação do agente causal, a alteração pode evoluir e culminar com a necrose das células epiteliais, principalmente nos casos de etiologia bacteriana (CARVALHO-JUNIOR et al., 2012). As causas de degeneração são inúmeras, considerando principalmente o aumento da temperatura escrotal em relação à corporal, além de processos inflamatórios agudos e crônicos, condições desfavoráveis de nutrição e hipovitaminoses (BICUDO et al., 2007).

Não foram observadas alterações testiculares relacionadas à hipoplasia, mas situações de estresse crônico, como as observadas em animais com enfermidades podais, podem resultar em inibição do eixo hipotalâmico-pituitária-gonadal. Um exemplo disso foi mostrado em um estudo com 59 bovinos leiteiros, com quadro clínico de claudicação. Foram feitos exames clínicos de claudicação, análise comportamental, dosagem hormonal e extração do 
leite e comprovou-se que a claudicação leva o organismo animal à um estresse crônico, porém sem afetar o ciclo estral. Entretanto, a claudicação pode provocar uma redução na frequência de expressão do comportamento sexual. E essa expressão, deve-se à limitação física, originada pelo próprio fator estressante (WALKER et al., 2010). Isso altera a liberação dos hormônios folículo estimulante (FSH) e luteinizante $(\mathrm{LH})$, alterando o efeito estimulatório na secreção de esteróides sexuais e modificando a fisiologia testicular (RAZDAN et al., 2001). Além disso, animais que diminuem a ingestão de alimentos durante a fase de crescimento podem apresentar diminuição no tamanho das gônadas (BONGSON et al., 1982; SALHAB et al., 2001).

Quanto às alterações na fisiologia testicular, MOURA et al. (2010), ao avaliar as características espermáticas de touros Nelore com dermatite digital, concluiram que as diferenças observadas nos parâmetros físicos espermáticos poderiam estar relacionadas à enfermidade podal. Porém, as características morfológicas seminais estudadas apresentaram-se dentro dos padrões estabelecidos pelo CBRA (1998). Com isso, constata-se que os animais estão aptos à reprodução, mas em relação a impotência coeundi, uma avaliação da gravidade da lesão podal e a capacidade de monta do touro, conforme SALVADOR et al. (2003), tornam-se fundamentais para se avaliar a influência das dermatites digitais na capacidade reprodutiva do macho bovino.

O estudo histomorfométrico dos testículos mostrou que os animais com dermatite digital apresentavam maiores medidas, especialmente em relação à altura do epitélio tubular, bem como menores valores na área da luz tubular quando comparados a animais sadios. De acordo com FRANÇA \& GODINHO (2003), a medida do diâmetro tubular é utilizada como indicador de atividade espermatogênica e função testicular, sendo considerado por SCOLFARO et al. (2003) um excelente indicador do desenvolvimento do epitélio seminífero.

Antes da puberdade, esse índice depende principalmente das células de Sertoli e indica se o epitélio seminífero está estimulado pelo FSH, tendo em vista que esse hormônio liga-se a receptores específicos dessas células (AGUIAR et al., 2006). Portanto, ao avaliar o diâmetro tubular, indiretamente também se avaliam as células de Sertoli (SCOLFARO et al., 2003).

Na puberdade, o diâmetro tubular também aumenta rapidamente. HOSHINO et al. (2002) verificaram que o diâmetro, a área e a altura do epitélio tubular seminífero apresentavam medidas maiores em cães adultos de diferentes raças.
Segundo os autores, esse achado ocorre devido ao estímulo da mitose e maior espermatogênese, gerando um epitélio extenso em número celular. Diante disso, também é possível que bovinos adultos jovens apresentem maiores medidas no que diz respeito a histomorfometria testicular, não sendo possível inferir sobre a influência do fator estresse crônico por dermatite digital como o desencadeador dos maiores valores de diâmetro e altura do epitélio tubular seminífero de bovinos.

A maioria dos campos testículares dos animais saudáveis apresentou área da luz tubular maior que aqueles dos bovinos com dermatite digital, embora sem diferença estatística entre os grupos. De acordo com KOIVISTO et al. (2008), a degeneração das células do túbulo seminífero, pode contribuir para a diminuição da produção de espermatides e alteração na qualidade espermática, bem como pode resultar em aumento da área da luz tubular, em decorrência da diminuição de células epiteliais.

\section{CONCLUSÕES}

Bovinos adultos jovens da raça nelore apresentam diferentes graus de degeneração testicular, bem como orquite intersticial crônica inespecífica, sem relação com a dermatite digital. As alterações histomorfométricas dos testículos desses bovinos não possuem relação com a enfermidade podal.

\section{REFERÊNCIAS}

AGUiAR, G. V.; ARAÚJO, A. A.; MOURA, A. A. A. Desenvolvimento testicular, espermatogênese e concentrações hormonais em touros Angus. Revista Brasileira de Zootecnia, v.35, n.4, 1629-1638, 2006.

ASSIS-NETO, A. C.; MELO, M. I. V.; CARVALHO, M. A. M.; MIGLINO, M. A.; OLIVEIRA, M. F.. Análise histométrica do desenvolvimento testicular de cutias (Dasyprocta aguti) criadas em cativeiros. Brazilian Journal of Veterinary Research and Animal Science, v.40, p.202-208, 2003.

BERGSTEN, C. Infectious diseases of the digits. In: GREENOUGH, P. R.; WEAVER, D. A. Lameness in Cattle. W.B. Saunders Company, Philadelphia, 3 ed, 1997. p.96-100.

BICUDO, S. D.; SIQUEIRA, J. B.; MEIRA, C. Patologias do sistema reprodutor de touros. Biológico, v.69, n.2, p.43-48, 2007.

BONGSON, T. A.; JAINUDENN, M. R.; SITIZHRAN, A. S. Relationship of scrotal, circumference to age, body weight and onset of spermatogenesis in goats. Theriogenology, v.18, p.513-524, 1982.

BOUCIF, A.; AZZI, N.; BOULKABOUL, A.; 
TAINTURIER, D.; NIAR, A. The testicular pathologies in rams of the Algerian local breed rembi clinical and histopathological classification. Asian Journal of Animal and Veterinary Advances, v.6, p. 831-837, 2011.

BOUSMAHA, F.; KHOUDJA, F. B. Comparative and pathological study of testis and epididymis in rams, bucks and bulls of Algeria. Asian Journal of Animal and Veterinary Advances, v.7, n.10, p. 950-959, 2012.

CARVALHO-JUNIOR, C. A.; MOUSTACAS, V. S.; XAVIER, M. N.; COSTA, E. A.; COSTA, L. F.; SILVA, T. M. A.; PAIXAO, T. A.; BORGES, A. M.; GOUVEIA, A. M. G.; SANTOS, R. L. Andrological, pathologic, morphometric, and ultrasonografic findings in rams experimentally infected with Brucella ovis. Small Ruminant Research, v.102, n.2, p.213-222, 2012.

CASTRO, G. R.; BRITO, L. A. B.; FIORAVANTI, M. C. S.; SILVA, L. A. F.; ARAÚJO, E. G.; ORLANDO, C. F. P.; FRANCO, L. G.; MOURA, M. I. Estudo anatomopatológico de lesões de dermatite digital em bovinos. Ciência Animal Brasileira, v.9, n.4, p.11591166, 2008.

CBRA. Manual para Exame Andrológico e Avaliação de Sêmen Animal. $2^{\text {nd }}$ ed. Colégio Brasileiro de Reprodução Animal, Belo Horizonte, MG. 1998. 49p.

CHAPLIN, S. J.; TERNENT , H. E.; OFFER , J. E.; LOGUE, D. N.; KNIGHT ,C. H. A comparasion of hoof lesions and behaviour in pregnant and early lactation heifer at housing. The Veterinary Journal, v.159, p.147153,2000

CHATE, S. C.; DIAS, R. A.; AMAKU, M.; FERREIRA, F.; MORAES, G. M.; COSTA NETO, A. A.; MONTEIRO, L. A. R. C.; LOBO, J. R.; FIGUEIREDO, V. C. F.; GONÇALVES, V. S. P.; FERREIRA NETO, J. S. Situação epidemiológica da brucelose bovina no Estado do Mato Grosso do Sul. Arquivo Brasileiro de Medicina Veterinária e Zootecnia, v.61, p.46-55, 2009.

COSTA, F. L. A.; SILVA, S. M. M. S.; NASCIMENTO, E. F. Pathologic evaluation of testis and epididymis of hairy rams in the semi-arid region of Piaui State. Arquivo Brasileiro de Medicina Veterinária e Zootecnia, v.59, p.1110-1116, 2007.

DEMIRKAN, I.; MURRAY, R. D.; CARTER, S. D. Skin diseases of the bovine digit associated with lameness. Veterinary Bulletin, v.70, p.149-171, 2000.

FARROW, C. S. Digital infection in cattle. Veterinary Clinics of North American Food and Animal Practice, v.15, p.411-423, 1999.

FERREIRA, P. M.; LEITE, R. C.; CARVALHO, A. U.; FACURY-FILHO, E. J.; SOUZA, R. C.; FERREIRA, M. G. Custo e resultados do tratamento de seqüelas de laminite bovina: relato de 112 casos em vacas em lactação no sistema free-stall. Arquivo Brasileiro de Medicina Veterinária e Zootecnia, v.56, n.5, p.589-594, 2004.

FONTANA, E. A.; WEAVER,W. D.; VAN KREY, H. P. Effects of various feeding regimens on reproduction in broiler-breeder males. Poultry Science, v.69, p. 209-16, 1990.

FRANÇA, L. R.; GODINHO, C. C. Testis morphometry seminiferous ephitelium cycle lenght and daily sperm production in domestic cats. Biology of Reproduction, v.68, p. 1554-1561, 2003.

HOCKING, P. M.; BERNARD, R. Effects of dietary crude protein content and food intake on the productionof semen in two lines of broiler breeder males. British Poultry Science, v. 38, n. 2, p.199-202, 1997.

HOSHINO, P.; NAKAGHI, L. S. O.; PACHECO, M. R.; LUI, J. F.; MALHEIROS, E. B. Morfometria dos túbulos seminíferos e ductos epididimários de cães e gatos provenientes de cirurgia de castração. Biotemas, v. 15, n. 1, p. 97-110, 2002.

KOIVISTO, M. B.; COSTA, M. T. A.; PERRI, S. H. V.; VICENTE, W. R. R. The effect of season on semen characteristics and freezability in Bos indicus and Bos Taurus bulls in the southeastern region of Brazil. Reproduction in Domestic Animals, v.42, n.4, p. 715726, 2008.

LADDS, P. W.; FOSTER, R. A. Male genital system. In: JUBB, K.V.F., KENNEDY, P.C., PALMER, N. (Eds.), Phatology of Domestic Animals. British, 2007. 3 rd, $5^{\text {a }}$ Ed, p. 565-618.

LEÃO, M. A.; SILVA, L. A. F.; JAYME, V. S.; SILVA, L. M.; MOURA, M. I.; BARBOSA, V. T. Aspectos epidemiológicos da dermatite digital bovina em duas propriedades produtoras de leite do estado de Goiás, Brasil. Ciência Animal Brasileira, v.10, n.4, p. 11351147, 2009.

LEONEL, F. P.; PEREIRA, J. C.; VIEIRA, R. A. M.; FREITAS, J. A.; DUTRA, A. R.; LIMA, A. V.; RIBEIRO, M. D.; COSTA, M. G. Exigências nutricionais em macronutrientes minerais ( $\mathrm{Ca}, \mathrm{P}, \mathrm{Mg}, \mathrm{Na}$ e $\mathrm{K}$ ) para novilhos de diferentes grupos genéticos. Revista Brasileira de Zootecnia, v.35, n.2, p.584-90, 2006.

MOURA, M. I.; GOULART, D. S.; ORLANDO, C. F. P.; FRANCO, L. G.; SILVA, O. C.; SILVA, L. A. F. Dermatite digital em bovinos da raça Nelore: Avaliação do ganho de peso, medidas testiculares e epididimárias no pós-operatório das lesões. Veterinária e Zootecnia (UNESP), v.17, p.239-249, 2010.

SALHAB, S. A.; ZARKAWI, M.; WARDEH, M. F.; ALMASRI, M. R, KASSEM, R. Development of testicular dimensions and size, and their relationship to age, body weight and parental size in growing Awassi ram lambs. Small Ruminant Research, v.40, p. 187-91, 2001.

SALVADOR, D. F.; ANDRADE, V. J.; VALE FILHO, V. R.; SILVA, A. S.; COSTA, E. V. Avaliação da libido de touros Nelore adultos em curral e sua associação com características andrológicas e desempenho reprodutivo a campo. Arquivo Brasileiro de Medicina Veterinária e Zootecnia, Belo Horizonte, v.55, n.5, p.588-593, 2003.

SAMPAIO, I. B. M. Estatística Aplicada à 
Experimentação Animal. Belo Horizonte: Fundação de Ensino e Pesquisa em Medicina Veterinária, 1998.

SCOLFARO, M. R.; CARDINALLI, I. A.; JUNIOR, G. G. A importância da análise histológica morfométrica gonadal na identificação da gônada disgenética. Arquivos Brasileiros de Endocrinologia \& Metabologia, v.47, n.2, p.128-134, 2003.

SILVA, L. A. F.; CARNEIRO, M. I.; FIORAVANTI, M. C. S.; MIRANDA, A. H.; BERNIS, W. O.; Uso da técnica de circuncisão com encurtamento do pênis para obtenção de rufiões equinos. Arquivo Brasileiro de Medicina Veterinária e Zootecnia, Belo Horizonte, v.47, n.6, p.789-798, 1995.

SILVA, L. A. F.; FIORAVANTI, M. C. S.; TRINDADE, B. R.; SILVA, O. C.; EURIDES, D.; CUNHA, P. H. J. C.; SILVA, L. M.; MOURA, M. I. Enfermidades digitais em vacas de aptidão leiteira: associação com mastite clínica, metrites e aspectos epidemiológicos. Pesquisa Veterinária Brasileira, v. 24, n.4, p.217-222, 2004.

TRANTER, W. P.; MORRIS, R. S. A case study of lameness in three dairy herds. New Zealand Veterinary Journal, Wellington, v.39, p.88-96. 1991.

VIU, M. A. O.; MAGNABOSCO, C. U.; FERRAZ, H. T.; GAMBARINI, M. L.; OLIVEIRA FILHO, B. D.; LOPES, D. T.; VIU, A. M. F. Desenvolvimento ponderal, biometria testicular e qualidade seminal de touros Nelore (Bos Taurus indicus) criados extensivamente na região Centro-Oeste do Brasil. Archives of Veterinary Science, v. 11, n. 3, p. 53-57, 2006.

WALKER, S. L.; SMITH, R. F.; JONES, D. N.; ROUTLY, J. E.; MORRIS, M. J.; DOBSON, H. The Effect of a Chronic Stressor, Lameness, on Detailed Sexual Behaviour and Hormonal Profiles in Milk and Plasma of Dairy Cattle. Reproduction in Domestic Animals, v.45, p. 109-117, 2010.

RAZDAN, P.; MWANZA, A. M.; KINDAHL, H.; HULTÉN, F.; EINARSSON, S. Impact of postovulatory food deprivation on the ova transport, hormonal profiles and metabolic changes in sows. Acta Veterinaria Scandinavica, v.42, n.1, p.45-55, 2001. 